\title{
Gender Differences in Neuroselective Current Perception Thresholds of the Trigeminal and Median Nerves
}

\author{
Nakazawa Yoshihito, DDS, Yatani Hirofumi*, DDS, PhD, \\ Minakuchi Hajime, DDS, PhD and Matsuka Yoshizou**, DDS, PhD \\ Department of Oral and Maxillofacial Rehabilitation, \\ Okayama University Graduate School of Medicine and Dentistry \\ * Department of Fixed Prosthodontics, Osaka University Graduate School of Dentistry \\ **Division of Oral Biology \& Medicine, UCLA School of Dentistry
}

Clinical significance

This study suggests that a gender-dependent difference in the pain threshold of peripheral nerves might not be a good explanation for the higher prevalence of orofacial pain in women.

\begin{abstract}
Purpose: The goal of this study was to ascertain gender differences in sensory thresholds by measuring current perception threshold (CPT) values of the trigeminal and median nerves in healthy individuals. Materials and Methods: CPT values were measured using a Neurometer ${ }^{\circledR}$ (Neurotoron, Baltimore, MD, USA) when electrical stimulation of 2,000, 250, or $5 \mathrm{~Hz}$ was applied to the left preauricular (trigeminal nerve) and thenar eminence (median nerve) regions in 20 healthy volunteers (10 males and 10 females with a mean age of 24.4 years). The day-to-day reliability of CPT was first verified in the trigeminal region by a test-retest method using an interclass correlation coefficient (ICC). The CPT values were then measured and statistically analyzed to evaluate whether the values were influenced by the electrical stimulation frequency and gender difference.

Results : Irrespective of the stimulation frequency, the ICC values were high $(2,000 \mathrm{~Hz}: 0.62,250 \mathrm{~Hz}$ : $0.80,5 \mathrm{~Hz}: 0.86)$, showing the high day-to-day reliability of CPT. The electrical frequency of the stimulation significantly influenced the CPT (ANOVA : $\mathrm{p}<0.0001$ ); the mean CPT value during 2,000 $\mathrm{Hz}$ stimulation was significantly higher than the value during 250 and $5 \mathrm{~Hz}$ stimulation (Bonferroni test $: \mathrm{p}<0.001)$. For median nerve stimulation, the mean CPT was lower for women than for men, but no significant gender difference was identified (ANOVA $: \mathrm{p}=0.11$ ). Conversely, gender exerted significant effects on CPTs (ANOVA $: \mathrm{p}=0.02$ ) for trigeminal nerve simulation. As to stimulation frequency, the mean $\mathrm{CPT}$ following $2,000 \mathrm{~Hz}$ stimulation of the trigeminal nerve was significantly lower for women than for men (Bonferroni test $: p<0.001$ ).

Conclusion: The results suggest that healthy women are more sensitive to tactile or pressure sensations compared to healthy men, and that the effects of gender differences on the sensory threshold can be ignored with pain-related nerve fibers such as $\mathrm{A} \delta$ and $\mathrm{C}$ fibers.
\end{abstract}

\section{Key words}

gender differences, current perception threshold, trigeminal nerve, median nerve

Corresponding author: Nakazawa Yoshihito

2-5-1, Shikata-cho, Okayama 700-8525, Japan

Tel : +81-86-235-6682, Fax : +81-86-235-6684

E-mail : nakazawa@md.okayama-u.ac.jp

Received on March 11, 2003/Accepted on July 4, 2003 


\section{INTRODUCTION}

A reduced pain threshold, caused by pain sensitization at the peripheral or central nerve level or loss of the pain suppression system, is believed to be involved in the onset of chronic maxillofacial pain ${ }^{1}$. However, ascertaining whether this reduced pain threshold occurs at the peripheral or central nerve level is not easy. To clarify the pathology of peripheral paresthesia and develop new therapeutic techniques, sensitivity thresholds of each peripheral sensory nerve fiber must be determined, and peripheral nerves with altered pain thresholds must be identified.

Epidemiological data have shown that chronic pain is more prevalent among women ${ }^{2}$, and one reason for this may be that pain sensitivity is greater in women ${ }^{3)}$. As part of an effort to identify causes of chronic facial pain by comparing sensory thresholds in healthy individuals to those in patients with chronic facial pain, the present study was conducted to evaluate gender differences in sensory thresholds by measuring neuroselective current perception thresholds of the trigeminal and median nerves in healthy individuals.

\section{MATERIALS AND METHODS}

\section{Reliability of Sensory Threshold Measurements}

Sensory thresholds were measured using a Neurometer ${ }^{\circledR}$ (Neurotoron, Baltimore, MD, USA), and reliability was investigated using the test-retest method. This neurometer is capable of non-invasively measuring current perception thresholds (CPTs). With three frequencies $(2,000,250$ and $5 \mathrm{~Hz}), \mathrm{CPT}$ s of $\mathrm{A} \beta, \mathrm{A} \delta$ and $\mathrm{C}$ fibers can be measured ${ }^{4}$. The subjects were 11 healthy individuals (6 men, 5 women ; mean age $25.1 \pm 1.2$ years) in whom clinical tests and medical inquiries revealed no mandibular or maxillofacial pain. Sufficient informed consent was obtained from all the subjects before starting the experiment. The automatic forced doubleblind technique was employed as a measurement protocol. Using the neurometer, CPT was measured on the facial skin about $10 \mathrm{~mm}$ anterior to the left external auditory canal (trigeminal nerve) twice with a 1-h interval after 4:00 PM. During this test, each subject was instructed to rest while wearing the sensor to eliminate the effects of different sensor positions on measurement reliability. Reliability was assessed by calculating an interclass correlation coefficient (ICC) for the first and second measurements, according to the standards of Landis and $\mathrm{Koch}^{5}$.

\section{Effects of Gender Differences on Sensory Threshold Measurements}

The subjects were 20 healthy individuals (10 men, 10 women; mean age $24.4 \pm 1.5$ years) in whom clinical tests and medical inquiries revealed no mandibular or maxillofacial pain. Sensory thresholds were measured according to the protocol above. Each subject was placed in the supine position in a quiet room, and all measurements were made between 4:00 PM and 6:00 PM. CPT was measured at two places: on the facial skin about $10 \mathrm{~mm}$ anterior to the left external auditory canal (trigeminal nerve), and at the left thenar prominence (median nerve). Two-way factorial ANOVA was used to ascertain the effects of stimulation frequencies and gender differences on CPT. When statistical significance was confirmed by ANOVA, the difference between mean CPT values was analyzed by a Bonferroni test at a significance level of $p=$ $0.05 / 3 \fallingdotseq 0.0167$

\section{RESULTS}

\section{Reliability of Sensory Threshold Measurements} ICCs for the trigeminal nerve following 


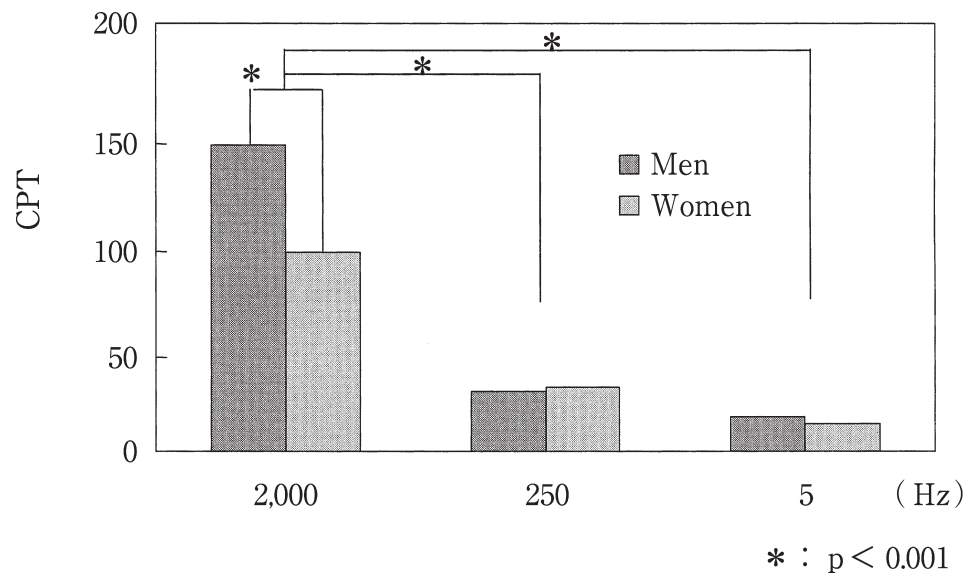

Fig. 1 CPTs of the trigeminal nerve

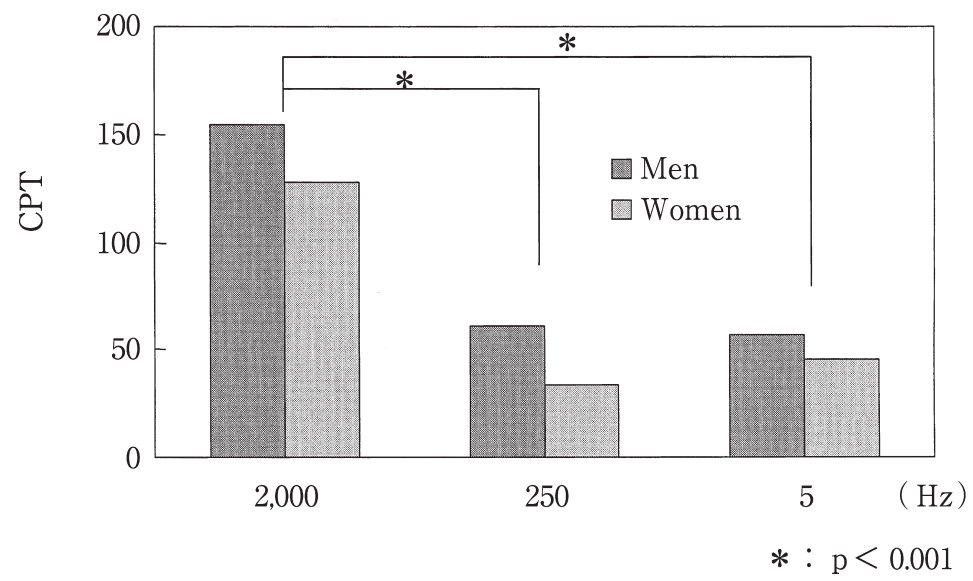

Fig. 2 CPTs of the median nerve

2,000, 250 and $5 \mathrm{~Hz}$ stimulation were $0.62,0.80$ and 0.86 , respectively. According to Landis and Koch's standards ${ }^{5}$, an ICC of $0.61-0.80$ is substantial, while $0.81-1.00$ is almost perfect. Reliability was therefore favorable for all three frequencies.

\section{Effects of Gender Differences on Sensory Threshold Measurements}

Stimulation frequency exerted significant effects on CPT (two-way ANOVA : principal effect, $\mathrm{p}<0.0001)$. For both trigeminal and median nerves, the mean CPT was significantly higher following $2,000 \mathrm{~Hz}$ stimulation than following 250 or $5 \mathrm{~Hz}$ stimulation (Bonferroni test $: \mathrm{p}<0.001$ ) (Figs. 1 and 2).

For median nerve simulation, the mean CPT for women was lower than that for men, but no significant gender difference was identified (two-way ANOVA : principal effect, $\mathrm{p}=0.11$ ) Conversely, gender exerted significant effects on CPTs for trigeminal nerve simulation (two-way ANOVA : principal effect, $p=0.02$ ). As to stimulation frequency, the mean CPT following 2,000 $\mathrm{Hz}$ stimulation of the trigeminal nerve was significantly lower for women than for men (Bonferroni test $: \mathrm{p}<0.001)$.

\section{DISCUSSION}

Since opportunities for patients with chronic orofacial pain to seek treatment at dental clinics for seeking treatment have increased recently, it is important for dentists, including prostho- 
dontists, to be able to deal with the patients properly. Chronic orofacial pain is probably due to peripheral and central sensitization or declined activities of the descending inhibitory system. Clinically, the prevalence of chronic orofacial pain is much higher in females than in males $^{3)}$, and it is partly explained by the higher pain sensitivity in females ${ }^{6}$. However, it has not been clarified whether there is actually a genderdependent difference in the pain threshold of peripheral nerves. Therefore, in the present study, we investigated gender differences in perception threshold of the trigeminal and median nerves.

The present study supports previous reports on the relationship between CPT and stimulation frequency or measurement $\operatorname{site}^{7)}$. In other words, higher stimulation frequency tended to result in greater CPT. In addition, the $\mathrm{CPT}$ of the trigeminal nerve was lower than that of the median nerve. As for the relationship between CPT and gender, the mean CPT following 2,000 $\mathrm{Hz}$ stimulation of the trigeminal nerve was significantly lower for women than for men. This suggests that, compared to healthy men, healthy women are more sensitive to tactile or pressure sensations, and that the effects of gender differences on the sensory threshold can be ignored with pain-related nerve fibers such as $\mathrm{A} \delta$ and $\mathrm{C}$ fibers. Although the causes of these gender differences could not be clarified based solely on results obtained from the trigeminal nerve, the present study suggests that gender differences should be considered when analyzing sensory thresholds in patients with chronic orofacial pain.

The possible gender difference of perception threshold in the orofacial region should be confirmed by future research.

Acknowledgements : A part of this study was presented at the 16 th Annual Convocation of the Japan Association of Cranio-Mandibular Orthopedics (September 15, 2002, Osaka) and the 108 th Scientific Meeting of the Japan Prosthodontic Society (October 11, 2002 , Nagoya). This study was partly supported by a Grantin-Aid for Scientific Research (B) (2) (No. 13470413) from the Ministry of Education, Culture, Sports, Science and Technology, Japan.

\section{REFERENCES}

1) Dickenson AHD. Pharmacology of pain transmission and control. In : Campbell JN, editor, Pain 1996-An Updated Review. Refresher Course Syllabus 113-121, Seattle : IASP Press, 1996.

2) Andersson HI. The epidemiology of chronic pain in a Swedish rural area. Qual Life Res 3 (Suppl 1): S 19-S 26, 1994.

3) Dao TT, LeResche L. Gender differences in pain. J Orofac Pain 14:169-84, 2000.

4) Katims JJ, Naviasky EH, Rendell MS et al. Constant current sine-wave transcutaneous nerve stimulation for evaluation of peripheral neuropathy. Arch Phys Med Rehabil 68:210-213, 1987.

5) Landis JR, Koch GG. The measurement of observer agreement for categorical data. Biometrics 3 : 159-174, 1977.

6) Isacson D, Bingefors K. Epidemiology of analgesic use : Gender perspective. Eur J Anaesthesiol 26 : 5-15, 2002.

7) Katims JJ, Patil A, Rendell M et al. Current perception threshold screening for carpal tunnel syndrome. Arch Environment Health 46 : 7-212, 1991. 